Please do not remove this page

RMIT

UNIVERSITY

\title{
Multi-wavelength variable drive-voltage modulator for use in high dynamic range photonic links
}

Ghorbani, Kamran; Scott, James; Mitchell, Arnan; Austin, Michael; Bui, Lam

https://researchrepository.rmit.edu.au/esploro/outputs/9921863272901341/filesAndLinks?institution=61RMIT_INST\&index=null

Ghorbani, K., Scott, J., Mitchell, A., Austin, M., \& Bui, L. (2007). Multi-wavelength variable drive-voltage modulator for use in high dynamic range photonic links. 2007 Asia-Pacific Microwave Conference Final Program, 1-4. https://doi.org/10.1109/APMC.2007.4555082

Published Version: https://doi.org/10.1109/APMC.2007.4555082

Repository homepage: https://researchrepository.rmit.edu.au

(c) 2007 IEEE. Personal use of this material is permitted. However, permission to reprint/republish this material for advertising or promotional purposes or for creating new collective works for resale or redistribution to servers or lists, or to reuse any copyrighted component of this work in other works must be obtained from the IEEE.

Downloaded On 2023/04/26 21:25:59 +1000 


\section{Multi-Wavelength Variable Drive-Voltage Modulator for use in High Dynamic Range Photonic Links}

\author{
J. Scott, K. Ghorbani, A. Mitchell, M. Austin and \\ L.Bui \\ School of Electrical and Computer Eng ineering \\ RMIT University \\ Melbourne, Australia \\ James.Scott@rmit.edu.au
}

\author{
T. Priest \\ Electronic Warfare and Radar Division \\ DSTO \\ Salisbury, Australia
}

\begin{abstract}
A novel technique for linearizing photonic links employing Mach-Zehnder modulators is presented. A truncated Fourier series representation of a linear transfer function is used to determine the modulator drive voltages $V_{\pi}$ and the optical power levels to be applied to a dual parallel modulator configuration. Simulation results are used to compare the dynamic range performance of the dual modulator configuration with a standard single modulator photonic link. The dual modulator configuration shows that improvements in compressive and spurious-free dynamic range can be achieved over the single modulator configuration, and that it is possible to further optimize the parameters to yield a significant improvement in spurious-free dynamic range without degrading the $1 \mathrm{~dB}$ compression point or the compressive dynamic range.
\end{abstract}

Keywords-linearization, photonic links, Mach-Zehnder modulators, high dynamic range links.

\section{INTRODUCTION}

The introduction of photonic systems in electronic warfare platforms has seen the improvement of dynamic range become a major research focus. High optical power and balanced detection has been used to maximize link gain while minimizing relative intensity noise (RIN) [1]. Linearity is also a significant factor limiting dynamic range and will be the focus of this paper.

The Mach-Zehnder modulator has an inherently non-linear transfer characteristic, which leads to the generation of unwanted harmonics and intermodulation products. These may then limit the dynamic range of the photonic link in which the modulator is embedded. An established technique for addressing this problem is to use multiple modulators to provide cancellation of unwanted distortion products $[2,3]$ or to use multiple polarizations within the one modulator to achieve cancellation [4]. In both cases the desired signals do not cancel.

The above-mentioned schemes have all been successful in significantly reducing harmonics or intermodulation products, but in all cases the cancellation is critically dependent upon the modulator characteristics. Bridges and Schaffner [5] have provided an excellent summary of the above linearization techniques and have presented link simulations using a simplified optical link model. They found that for systems

This work was sponsored by DSTO, R\&D contract \#4500310001. employing linearization, the distortion products no longer follow a simple exponential relationship to the RF drive level. Therefore, parameters such as the third-order intercept point cannot be simply calculated as the third-order products do not follow the typical 3:1 level increase versus RF input power level. Situations can also arise where a particular distortion product $\left(I M_{3}\right.$ or $2^{\text {nd }}$ harmonic) may cross the output noise level a number of times, with the slope of the curve being different at different locations. They therefore proposed that the dynamic range should be specified as a Spurious-free Dynamic Range (SFDR), being the difference in input RF power level between the point where the signal output level is equal to the output noise level and the point where the first distortion product is equal to the output noise level.

It should be noted that all of the schemes reported above employed modulators with the same $V_{\pi}$, and the biasing of the modulators and the selection of $V_{\pi}$ were not used as design variables. Only the RF and optical power levels were used as design variables. Also, it has been assumed that the modulators are biased at quadrature, thus ensuring suppression of the second harmonic.

All of the cited analyses have also assumed operation at a single frequency. It is known that the modulator characteristics, and in particular the modulator drive voltage, may vary significantly with frequency. An investigation [6] has been published on the impact of bandwidth on optimal dynamic range configurations.

As concluded in [4], optimal dynamic range performance is very sensitive to the characteristics of the modulators used. In this paper we use a single modulator device type with multiple optical wavelengths. One issue that must be considered is bias point variation with wavelength. Even small deviations of the bias point from quadrature may result in the emergence of significant second harmonic distortion. A technique to optimize the biasing of a single Mach-Zehnder modulator has been reported by Dubovitsky et al. [7]. In this paper, the aim was to ensure that the bias point of the modulator was optimized across a range of wavelengths for WDM applications. The technique does not, however, improve the $I_{3}$ performance of the modulator.

We propose a similar approach to [5], but instead of adjusting the RF attenuation, we propose varying the 
modulator drive voltage $\left(V_{\tau}\right)$. Our novel implementation utilizing WDM techniques allows simultaneous operation with multiple $V_{\pi}$. A dual modulator scheme is compared with a conventional single modulator photonic link and then results are presented for an optimized dual modulator configuration.

\section{PHOTONIC LINK ANAL YSIS}

A diagram of a standard photonic link is presented in Figure 1. The modulator is driven with two signal generators, which are combined using an RF combiner and the intermodulation products are measured on a spectrum analyzer. Typical parameters for the photonic link are given in Table I.

TABLE I. TYPICAL PARAMETERS FOR THE STANDARD PHOTONIC LINK

\begin{tabular}{|c|l|c|}
\hline Parameter & \multicolumn{1}{|c|}{ Description } & Value \\
\hline$P_{\mathrm{op}}$ & Optical power & $10 \mathrm{dBm}$ \\
\hline$R I N$ & Relative intensity noise & $-160 \mathrm{~dB} / \mathrm{Hz}$ \\
\hline$\alpha_{\mathrm{m}}$ & Modulator optical loss & $4.7 \mathrm{~dB}$ \\
\hline$V_{\pi}$ & Modulator drive voltage & $4.5,13.5 \mathrm{~V}$ \\
\hline$R_{\mathrm{mod}}$ & Modulator electrode impedance & $40 \Omega$ \\
\hline$\alpha_{\mathrm{op}}$ & Optical loss in transmission & $10 \mathrm{~dB}$ \\
\hline$G_{\mathrm{op}}$ & Gain of the EFDA & $10 \mathrm{~dB}$ \\
\hline$\Re$ & Photodetector responsivity & $0.8 \mathrm{AW}$ \\
\hline$R_{\mathrm{o}}$ & Photodetector load impedance & $50 \Omega$ \\
\hline$B W$ & Noise electrical bandwidth & $1 \mathrm{MHz}$ \\
\hline
\end{tabular}

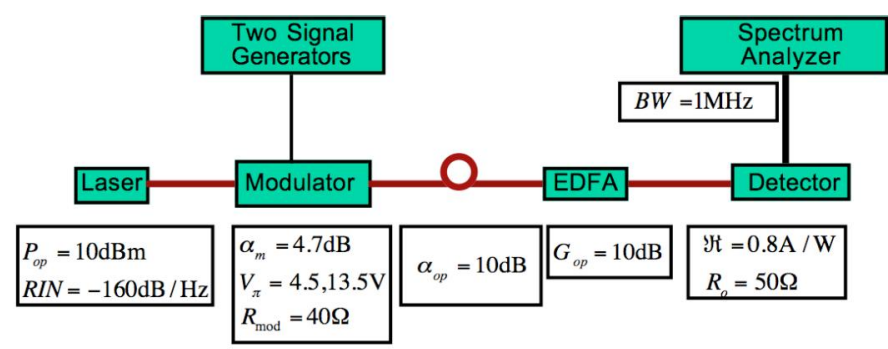

Figure 1. Schematic of standard photonic link with system parameters labelled.

Agilent-EEsof's Advanced Design System (ADS) was used to perform nonlinear analyses on the various link configurations. In the analyses, the optical intensity was modeled as voltage. A harmonic balance analysis with the nonlinear noise analysis option activated was used to simulate the link and include the various noise sources within the photo detector and the optical amplification. The electrical bandwidth was used for the noise calculations.

An initial analysis using a $V_{\pi}$ of $4.5 \mathrm{~V}$ is shown in Figure 2. This link yielded an input $1 \mathrm{~dB}$ compression point of $13 \mathrm{dBm}$, a compressive dynamic range (CDR) of $75 \mathrm{~dB}$ and a SFDR of $56 \mathrm{~dB}$ (SFDR is calculated throughout the paper for a noise electrical bandwidth of $1 \mathrm{MHz}$ ). To investigate the impact of increasing the drive voltage, the system was remodeled with a $V_{\pi}$ of $13.5 \mathrm{~V}(3 \times 4.5)$. As expected, the input $1 \mathrm{~dB}$ compression point increased to $22 \mathrm{dBm}$, but the CDR and SFDR have remained constant at $75 \mathrm{~dB}$ and $56 \mathrm{~dB}$ respectively. These results confirm that adjusting the modulator $V_{\pi}$ voltage does not alter the inherent dynamic range of the photonic link. The use of two identical modulators in parallel will now be discussed as a means of linearizing the photonic link.

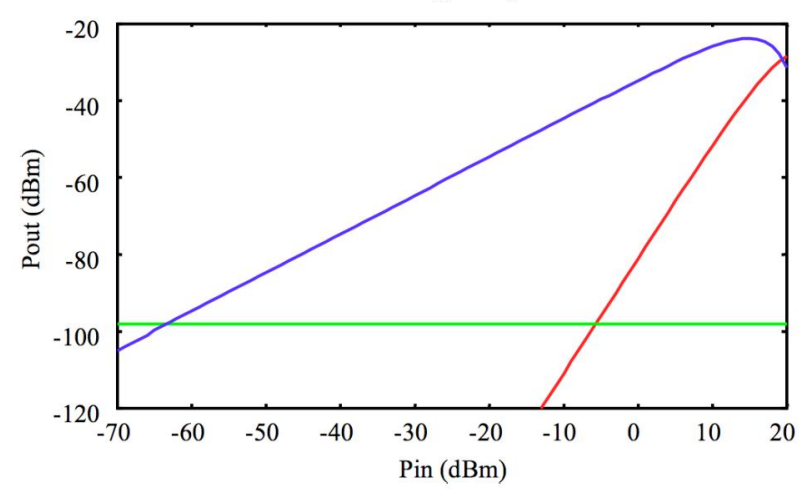

Figure 2. The modulated power out as a function of input power for the first harmonic and $M_{3}$ products of the photonic link presented in Figure 1 and

Table I with a $V_{\pi}$ voltage of $4.5 \mathrm{~V}$. The noise floor is also presented (calculated for a $1 \mathrm{MHz}$ noise electrical bandwidth).

\section{LINEARIZATION TECHNIQUE}

The dynamic range of the Mach-Zehnder optical modulator is limited by the fact that when it is biased at quadrature, its transfer function is only approximately linear for small input signals. The transfer function is actually sinusoidal, so modulation with a large RF signal will lead to nonlinear effects.

In order to achieve a linear response, a combination of multiple modulators in parallel can be used to approximate a linear transfer function. Consider a triangular wave as an example of a linearized response. This function can be decomposed into a Fourier series given by:

$$
F(x)=\sum_{N=0}^{\infty} 1 /(2 N+1)^{2} \sin ((2 N+1) x)
$$

Taking the first two terms of this series we have

$$
F(x)=\sin (x)+(1 / 9) \sin (3 x)
$$

To implement such a linearized function with two dual parallel modulators, we can use the configuration shown in Figure 3. Here one modulator has a $V_{\pi}$ voltage of $4.5 \mathrm{~V}$ while the other has a $V_{\pi}$ voltage of $3 \times 4.5=13.5 \mathrm{~V}$. The optical power of the low $V_{\pi}$ modulator is (1/9) that of the high $V_{\pi}$ voltage device. Note that the ratio of the two optical powers and the two $V_{\pi}$ voltages correspond to the ratios of the multipliers of the $\sin$ functions and the coefficients of $x$ respectively in (2). Use of additional modulators with a subsequent increase in the number of wavelengths used allows additional terms of the Fourier series to be realized. A greater degree of linearization (at the expense of increased complexity) is then possible. 


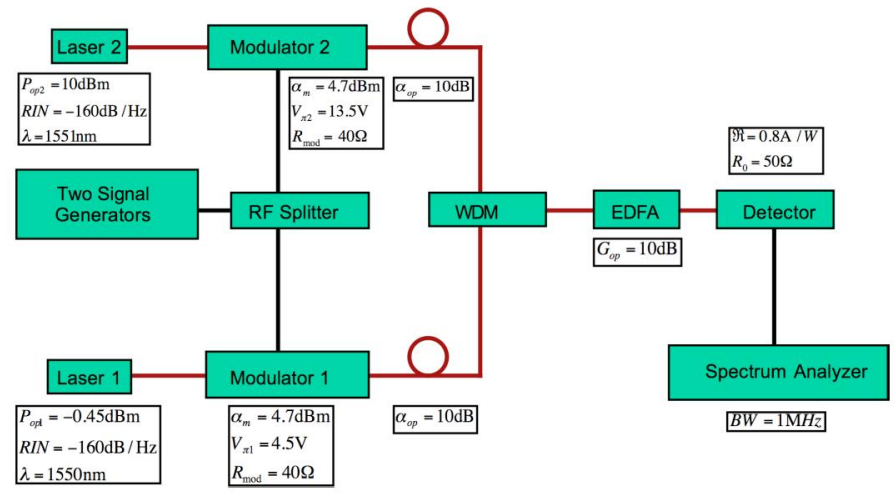

Figure 3. Schematic of dual parallel linearized photonic link.

\section{RESULTS}

\section{A. Linearized Link with Two Modulators}

The system of Figure 3 was simulated using ADS and the response is given in Figure 4 . The input $1 \mathrm{~dB}$ compression point, the CDR and the SFDR are $22 \mathrm{dBm}, 75 \mathrm{~dB}$ and $46 \mathrm{~dB}$ respectively. Comparing these results with those for the standard photonic link discussed earlier, we can see that in addition to the decrease in dynamic range, a null occurs in the third order intermodulation $\left(I M_{3}\right)$ response. The location of this null can be exploited to improve the $M_{3}$ response as will be shown in the next section.

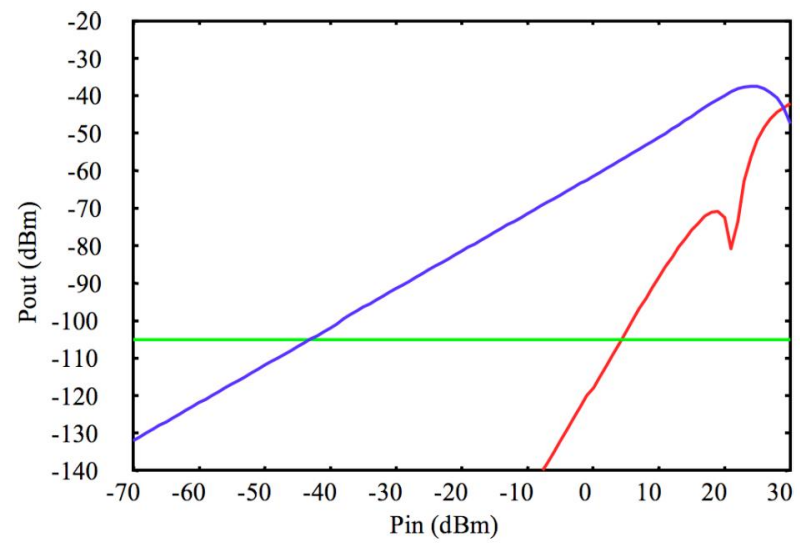

Figure 4. The detected RF power as a function of input RF power for the first harmonic and $I M_{3}$ products of the two modulator linearized photonic link.

\section{B. Optimally Linearized Link with Two Modulators}

The location of the null observed in the $\mathrm{IM}_{3}$ response is related to the choice of triangular waveform for the linearized response. To optimally improve the dynamic range of the system, the parameters of the dual parallel modulator configuration can be adjusted such that the null and nearby lobe are just below the noise floor of the system and thus the dynamic range is determined by the second lobe of the $I M_{3}$ response

This optimization was the topic of investigation reported by Bridges and Schaffner [5]. They found that the optimal configuration is a factor of 2.7 in $V_{\pi}$ and a factor of $(2.7)^{3}$ in optical power. The optimized system results from the ADS simulations are shown in Figure 5 . The input $1 \mathrm{~dB}$ compression point remains at $22 \mathrm{dBm}$ and the $\mathrm{CDR}$ at $75 \mathrm{~dB}$ (the values obtained for the dual parallel non-optimized link) but the SFDR has increased significantly to $68 \mathrm{~dB}$.

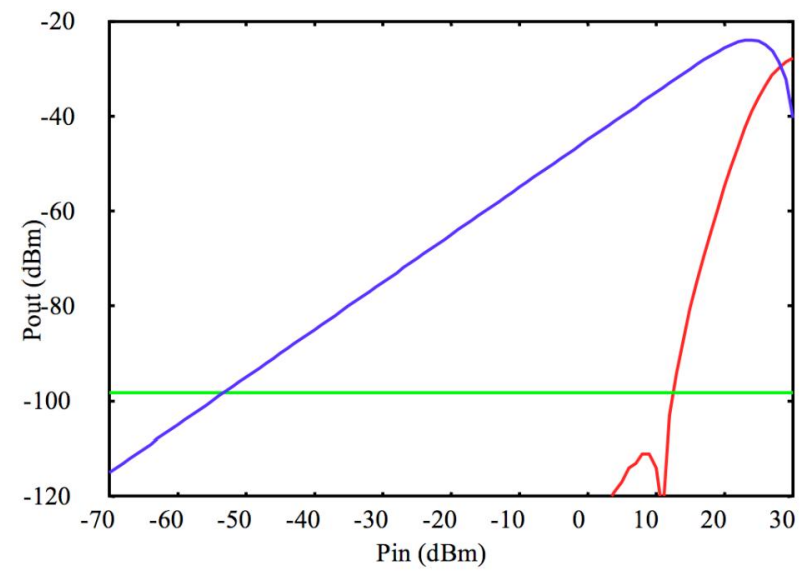

Figure 5. The detected RF power as a function of input RF power for the first harmonic and the $I M_{3}$ responses of the optimised two-modulator linearized photonic link, with $V_{\pi}$ voltages of $4.5 \mathrm{~V}$ and $12.5 \mathrm{~V}$ and optical powers of $10 \mathrm{dBm}$ and $-3 \mathrm{dBm}$ respectively.

By comparing Figures 4 and 5 it is evident that a significant improvement in SFDR can be achieved, which agrees with the findings reported in [5]. In a practical system, the optical power can be controlled dynamically and remotely by adjusting the laser power delivered to each modulator. Trimming of the $V_{\pi}$ voltages remotely is much more difficult and it is expected that the $V_{\pi}$ voltages of the two modulators will vary with frequency. This will be further exacerbated by the RF power split between the two modulators not being balanced for all frequencies, leading to a further varying of the $V_{\pi}$ voltages.

Figure 6 presents the response of the two-modulator system with $V_{\pi 2}$ adjusted from 12.5 to $17.5 \mathrm{~V}$. Here it can be seen that increasing the $V_{\pi}$ of modulator 2 shifts the null in the third harmonic towards higher input powers and raises the nearby lobe above the noise floor. The dynamic range thus reduces (from $68 \mathrm{~dB}$ to $55 \mathrm{~dB}$ ) approaching the un-optimized situation of Figure 4 .

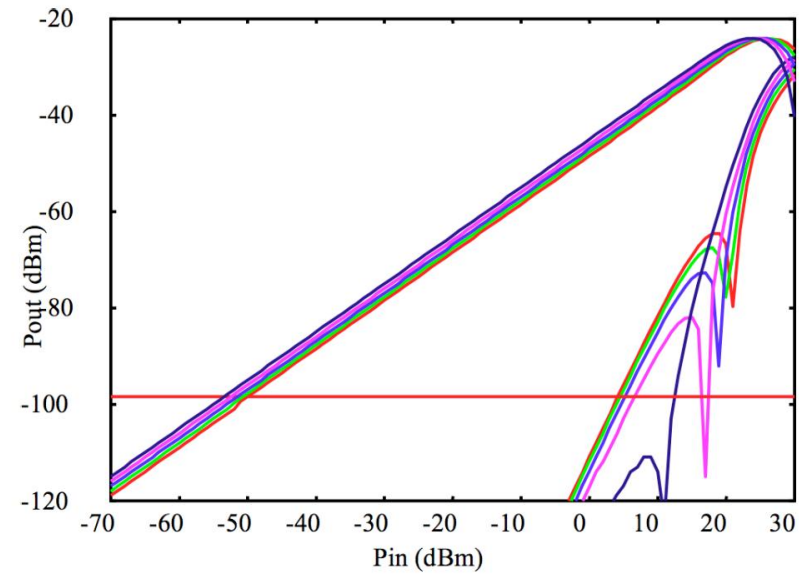

Figure 6. The detected RF power as a function of input RF power for the first harmonic and the $M_{3}$ responses of the optimized two-modulator linearized photonic link. $V_{\pi}$ voltages $V_{\pi 1}=4.5 \mathrm{~V}, V_{\pi 2}$ ranges from 12.5 to $17.5 \mathrm{~V}, P_{\mathrm{op} 2}=10 \mathrm{dBm}$ and $P_{\mathrm{op} 1}=-3 \mathrm{dBm}$ 
Figure 7 presents the response of the dual modulator linearized system with $V_{\pi 1}$ adjusted from 4.5 to $6.3 \mathrm{~V}$. For even a slight increase of the $V_{\pi}$ of modulator 1 with respect to modulator 2 , the null in the third harmonic disappears and the dynamic range degrades rapidly (from $68 \mathrm{~dB}$ to $60 \mathrm{~dB}$ ).

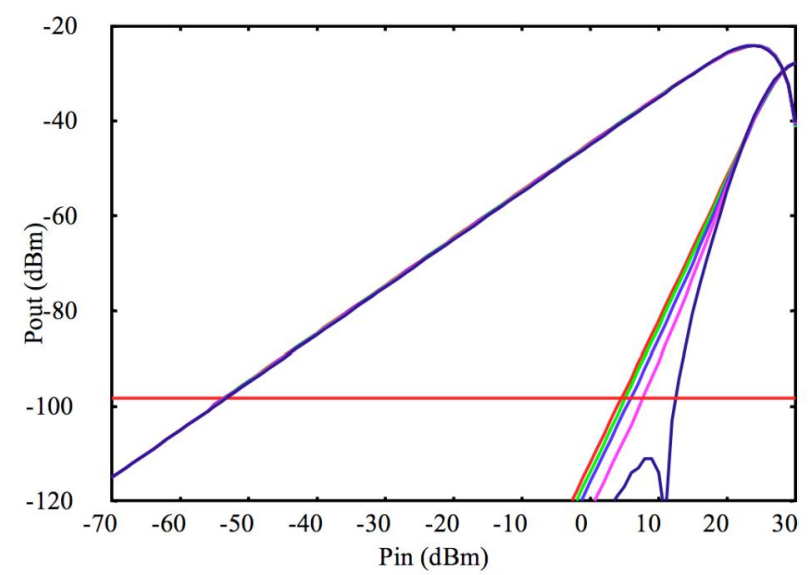

Figure 7. The detected RF power as a function of input RF power for the first harmonic and the $I M_{3}$ responses of the optimized two-modulator linearized photonic link. $V_{\pi}$ voltages $V_{\pi 1}$ ranges from 4.5 to $6.3 \mathrm{~V}$,

$$
V_{\pi 2}=12.5 \mathrm{~V} . P_{\mathrm{op} 2}=10 \mathrm{dBm} \text { and } P_{\mathrm{op} 1}=-3 \mathrm{dBm} \text {. }
$$

It can be concluded from this investigation that the $V_{\pi}$ voltages of the two modulators must be precisely balanced. This suggests that the bandwidth of such a system will be limited by the RF components used to deliver the signal to the modulators and also by the differences in the modulators themselves.

\section{CONCLUSIONS}

A model in Agilent-EEsof ADS has been constructed that is capable of modeling the dynamic range of various photonic link configurations including single and dual-modulator linearized configurations. The dynamic range of a single photonic link has been accurately modeled and the dynamic range of a dual parallel modulator has been modeled and it has been shown that the third harmonic can be nulled at a particular input RF power level, thereby greatly enhancing the SFDR. The sensitivity of the optimized configuration has been investigated and it has been shown that even small changes in $V_{\pi}$ voltage can significantly affect the resulting dynamic range. This will have a significant impact on the operating bandwidth of such linearized photonic links. It is recommended that further work be undertaken to investigate the possibility of recovering degraded dynamic range performance for wideband applications through fine-tuning of the optical power of each modulator. Experimental results for the dual modulator systems will be presented at the conference.

\section{REFERENCES}

[1] Newberg, I.L., "High-performance lossless RF fiber optic link", IEEE International Conference on Phased Array Systems and Technology, pp. $375-378,2000$.

[2] S.K. Korotky and R.M. de Ridder, "Dual parallel modulation schemes for low-distortion analog optical transmission", IEEE Journal on
Selected Areas in Communications, Vol. 8, No. 7, pp. 1377-1381, Sept. 1990.

[3] J.L. Brooks, G.S. Maurer and R.A. Becker, "Implementation and evaluation of a dual parallel linearization system for AM-SCM video transmission", Journal of Lightwave Tech., Vol. 11, No. 1, pp. 34-41, Jan. 1993.

[4] L.M. Johnson and H.V. Roussell, "Reduction of intermodulation distortion in interferometric optical modulators", Optical Letters, Vol. 13, No. 10, pp. 928-930, Oct. 1988.

[5] W.B. Bridges and J.H. Schaffner, "Distortion in linearized electrooptic modulators", IEEE Trans. on Microwave Theory and Tech., Vol. 43, No. 9, pp. 2184-2197, Sept. 1995.

[6] U.V. Cummings and W.B. Bridges, "Bandwidth of linearized electrooptic modulators", Journal of Lightwave Tech., Vol. 16, No. 8 , pp. 1482-1490, Aug 1998.

[7] S. Dubovitsky et al., "Analysis and improvement of Mach-Zehnder Modulator linearity performance for chirped and tunable optical carriers", Journal of Lightwave Tech., Vol. 20, No. 5, pp. 886-891, May 2002 . 